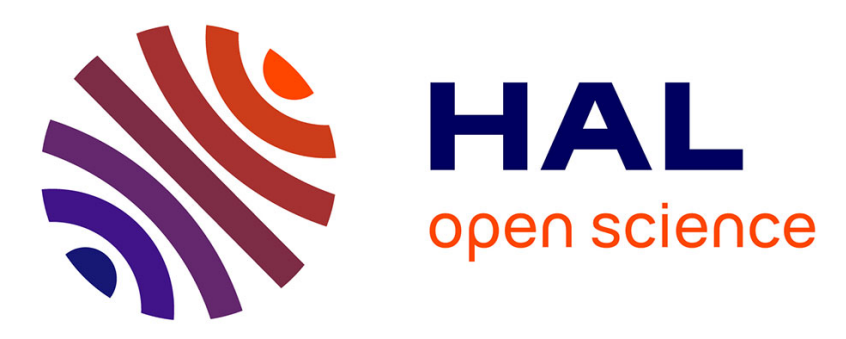

\title{
Aligning Business Process Models and Domain Knowledge: A Meta-Modeling Approach
}

Samira Si-Said Cherfi, Sarah Ayad, Isabelle Comyn-Wattiau

\section{To cite this version:}

Samira Si-Said Cherfi, Sarah Ayad, Isabelle Comyn-Wattiau. Aligning Business Process Models and Domain Knowledge: A Meta-Modeling Approach. European Conference on Advances in Databases and Information Systems, Sep 2012, Poznan, Poland. pp.45-56, 10.1007/978-3-642-32741-4_5 . hal00764068

\section{HAL Id: hal-00764068 \\ https://hal.science/hal-00764068}

Submitted on 13 Dec 2012

HAL is a multi-disciplinary open access archive for the deposit and dissemination of scientific research documents, whether they are published or not. The documents may come from teaching and research institutions in France or abroad, or from public or private research centers.
L'archive ouverte pluridisciplinaire HAL, est destinée au dépôt et à la diffusion de documents scientifiques de niveau recherche, publiés ou non, émanant des établissements d'enseignement et de recherche français ou étrangers, des laboratoires publics ou privés. 


\title{
Aligning Business Process Models and Domain Knowledge: A Meta-Modeling Approach
}

\author{
Samira Si-Said Cherfi ${ }^{1}$, Sarah Ayad ${ }^{1}$, Isabelle Comyn-Wattiau ${ }^{2}$ \\ ${ }^{1}$ CEDRIC-CNAM, 292 Rue Saint Martin, F-75141 Paris Cedex03 \\ 2 CEDRIC-CNAM and ESSEC Business School \\ samira.cherfi@cnam.fr, ayad.sarah87@gmail.com,wattiau@cnam.fr
}

\begin{abstract}
In recent years the problems related to modeling and improving business processes have been of growing interest. Indeed, companies are realizing the undeniable impact of a better understanding and management of business processes (BP) on the effectiveness, consistency, and transparency of their business operations.In this paper we propose to support this modeling effort with an approach that uses domain knowledge to improve the semantic quality of BP models. This approach relies on domain ontologies as a mean to capture domain knowledge and on meta-modeling techniques. The main contribution of this paper is threefold: 1) the meta-models describing both a domain ontology and a BP model are described, 2) the alignment between the concepts of both meta-models is defined and illustrated, 3) a set of OCL mapping rules is provided. A simple case study illustrates the process.
\end{abstract}

Keywords: Domain knowledge, Domain ontology, Semantic quality, Business process modeling, Quality improvement.

\section{Introduction}

- Business Process models are conceptual models supposed to give a complete description of the underlying business processes.This tuning requires a better understanding and an effective management of BP. BP modeling is difficult, it is an expert task that needs to be performed by trained experts. And, what about quality? Quality can be defined as the total of properties and characteristics of a product or service that are relevant for satisfying specific and obvious requirements [1]. The business process modeling approaches share many similarities with conceptual modeling activities, but are much more complex [2].

We propose to assist the modeling activity with a quality centered approach that aims to exploit the domain knowledge. The domain knowledge in Information Systems discipline refers to knowledge provided by both methods and application domain [24]. In our approach we propose to exploit domain ontologies knowledge with alignment rules to identify similarities between BP models and domain ontologies elements. The aim is to improve the semantic completeness and expressiveness of BP models according to domain knowledge contained in the ontologies. 
This paper is organized as follows. State of the art is described briefly in Section 2. The overall approach of our semantic is broadly described in the third section. The meta-models structuring both BP models and domain ontologies are described in detail in Section 4. Section 5 is dedicated to alignment rules. Finally Section 6 concludes and describes future research.

\section{State of the art}

A Business Process (BP) is a set of related activities that transform an input to create an output with added values [3]. Experts in information systems and professionals agree that the success of a company depends particularly of a good understanding of business processes [4]. To make a business process model understandable, reliable, and reusable it is important to ensure its quality. Several approaches that work in this direction exist in the literature. We have classified them into three categories:

1. Approaches focused on improving BP methods of analysis and design: improving the process development improves the quality of products. we can mention [5] where the authors propose a set of guides to improve clarity, comprehensibility. Other authors focus on improving the comprehensibility of models [6].

2. Process quality measurement: considers the quality level of business processes and their execution. We categorize the research on simulation and control of process as in [8]. In [9], the authors present and discuss several techniques for the analysis of processes during execution such as verification.

3. Process model quality measurement: Our focus is in this category that addresses the quality from the point of view of its evaluation and improvement. In [10], the authors mention the most important five measures: coupling, cohesion, complexity, modularity, and finally the size. [11] propose an approach based on GQM method (Goal-Question-Metric). One of the characteristics that has been the subject of several proposals is the complexity $[13,22]$. However, these studies are based primarily on structural characteristics of processes and their models.

In conclusion, our analysis of the state of the art leads us to argue that the quality of BP model is mainly addressed in terms of structural and syntactic and rarely in terms of semantics. In the remainder of this paper, we present our approach which aims to go a step forward into a semantic quality based approach of BP model.

\section{The overall approach for semantic quality improvement}

Modeling activity in general and BP modeling in particular are creative activities conducted by modelers using a given notation or modeling language. The result is of course highly dependent on the modeler experience in the notation practice, on his/her interpretation of the reality. This explains the fact that several correct but different 
models can usually be generated from the same reality. However, these models are supposed to be faithful representations of the reality. Thus the definition of quality requirements for these models is, in fact, a mean to evaluate this modeling activity and ensure a better result. The semantic quality measures the degree of correspondence between the model and the domain and it is related to both completeness and validity of the models; here the BP models [16].

To improve the quality of models produced, several approaches are possible: assistance in the development process phase by generic methodological guides from experience, measurement of the specifications quality, reusing approved specifications fragments etc. In this paper, we propose to exploit knowledge of field, which are supposed to reflect the knowledge shared by a community of actors, in order to improve the quality of process models.

Our approach relies on the process having as input point the business process model to be evaluated and a domain ontology representing business knowledge and rules of the underlying problem domain.

The steps of the process are the following:

- Discovering similarities between input BP model and domain ontology: this is based on a set of alignment rules at both syntactic and semantic levels.

- Evaluating semantic quality includes measuring a value of quality according to quality metrics.

- Improving semantic quality: An originality of our work is to integrate the quality improvement within the proposed approach.

1. Identifying Model-Ontology similarities: In the first step, the approach consists in discovering the mappings between business process model elements and the domain ontology elements. To make these alignment rules generic and independent of both the BP modeling notation and the ontology implementation language, we have defined two meta-models namely a BP meta-model and an ontology metamodel presented in detail in Section 4. Once these similarities identified they serve as input for both semantic quality evaluation and improvements activities. In this paper we mainly focus on this alignment activity.

2. Evaluating Semantic Quality : Semantic quality expresses the degree of correspondence between the information expressed within a model and the domain that is modeled. In order to evaluate the semantic quality we have identified a set of what we call quality deficiencies such as incompleteness and ambiguity. These deficiencies result from modeling choices producing models that do not cover the intended requirements or with low expressiveness. Such models lead to inadequate systems due to incompleteness or to misunderstanding during their implementation.

3. Quality Improvement : The quality improvement activity consists in suggesting to the analyst or the quality expert a set of improvement guidelines to improve the quality of their models. Again, this step uses the domain knowledge to generate improvement actions. This means that the completeness and even the relevance of these guides rely partly on the quality of the domain ontology but this aspect is out of the scope of our approach. 
This article focuses on discovering similarities between BP models and domain ontologies. We will however provide some examples of improvements without detailing the mechanism leading to generate them in Section 5, dedicated to the illustration of the approach.

\section{Ontology and process model meta-models.}

In order to identify similarities between knowledge contained in the ontology and the one represented by the BP model, our approach relies on alignment. To ensure the generality of these rules, we have chosen to define them at a meta-modeling level. Hence, the first contribution is the construction of meta-models representing ontologies and BP models.

\subsection{Business Process Meta-model}

The meta-model provides a synthetic vision of concepts used independently of specific notations helping in the understandability of models. And since we consider that domain knowledge contains also knowledge embedded in methods and consequently in notations, we will use meta-models to integrate completeness, validation and correctness rules defined by BP notations to enrich our actual vision of domain knowledge.

The meta-model defined in this section was constructed as a synthesis of a selection of concepts proposed by several authors and according to several notations and more specifically the work presented in $[17,18]$.

A business process model is composed of flows of objects and connectors. A flow object can be an event, an activity or a gateway [19]. An event that occurs is a fact and impacts the progress of a process. An activity can be an atomic task if it is not decomposable or a process if it is complex and has a visible structure. A gateway is a mechanism that can manage the convergence or divergence of activities flow. A connecting element can be an association, a simple link between two concepts. A sequence which defines an execution order of activities. And a message flow which is used to represent exchange of information between two participants in the process.

Activities refer to resources. A resource is a concept which includes abstract concepts such as the human agent responsible for execution of the activity and information produced or consumed by it. The exact role of the resource in the process is explained by the concept of role. Figure 1 shows an example of BP model from a "Mission order" case study. The example uses the Eriksson and Penker notation for Business process modeling [17].

\subsection{Ontology Meta-model}

The ontology meta-model allows representing domain ontologies using the same concepts independently of the language for their implementation. In our approach, we consider an ontology as a set of classes and relationships. This vision is largely 
adopted. We distinguish between three types of concepts of type class: actor, action and artifact.

- An actor is an independent entity, able to perform actions.

- An action represents the execution of an action.

- An artifact is an inanimate object incapable of performing an action. An artifact may represent an information or an abstract concept.

However, most of meta-models take into account two kinds of relationships, namely inheritance and structural relationships. For the needs of our approach we adapted the classification of relationships proposed by [22]. This classification offers several types of relationships allowing us to characterize precisely the nature of links between concepts.

Relations are first decomposed into three categories:

- Status: represents relationships that may be structural (inheritance, instantiation), influence (control, creation, destroy), or temporal (follow, precede).

- Change of status: reveals the occurrence of remarkable events.

- Interaction: represents short-term relationships between entities. Several semantic relations are defined for interactions such as communication, observation, execution, etc.

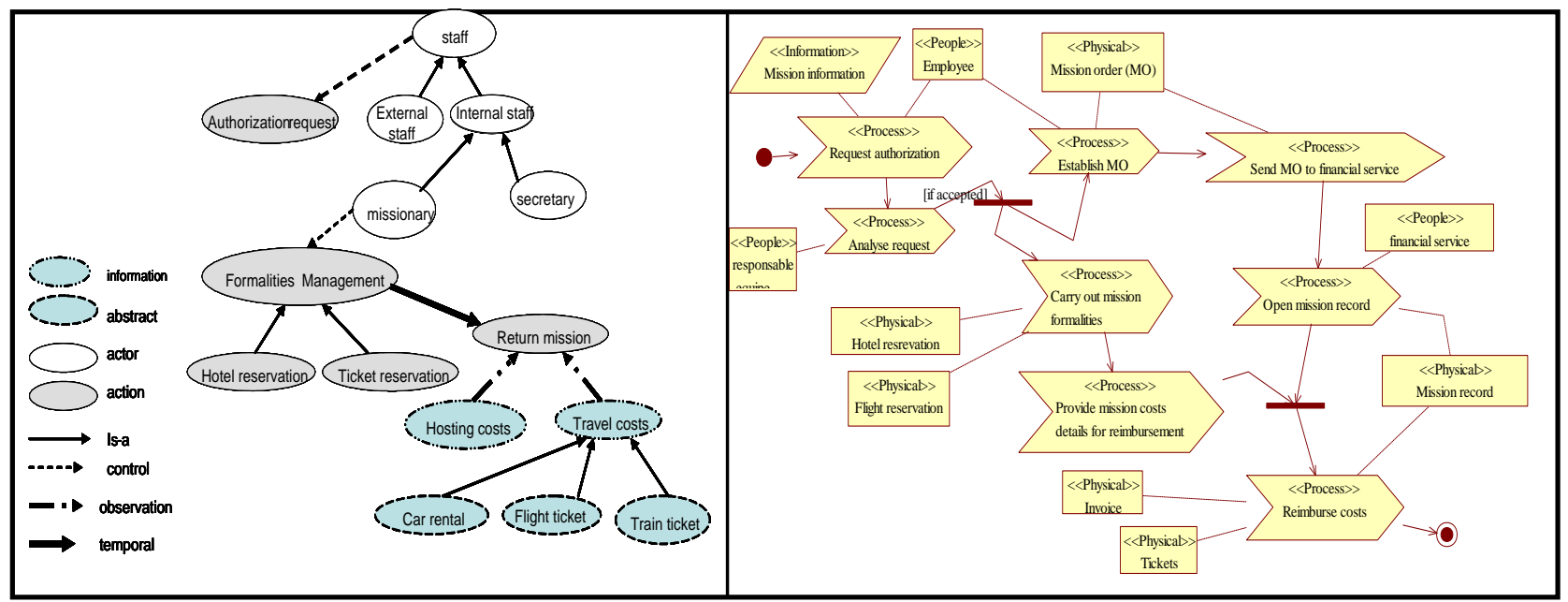

Fig. 1 Instantiation of the BPM meta model and of the Ontology meta-model

Figure 1 shows an example of a domain ontology. This is an extract from the ontology "mission plan".

\section{$5 \quad$ Mapping Process Model and Ontology Meta-Models}

Thanks to the precise categorization of concepts in both ontology and process model meta-models we are likely able to predefine some concepts correspondences allowing the mapping of the domain ontology concepts with the PM concepts. 
We have defined two kinds of mapping, namely type-based mapping and semantics-based mapping.

\subsection{Type-based Mapping Rules}

This mapping involves the types of concepts in order to establish correspondences between the concepts at the meta-level. These correspondences allow reconciliation based on the types of concepts independently of their meaning. These rules are still essential to avoid typing errors. An extract of predefined meta-model concepts mappings is given in Table 1.

Table 1. Concept alignement

\begin{tabular}{l|l}
\hline $\begin{array}{l}\text { BP model } \\
\text { meta-model concept }\end{array}$ & $\begin{array}{l}\text { Domain Ontology } \\
\text { meta-model concept }\end{array}$ \\
\hline People resource & Actor \\
Abstract resource & Abstract \\
Information resource & Knowledge \\
Process / activity & Action \\
\hline
\end{tabular}

Table 2. Relation alignement

\begin{tabular}{l|l}
\hline $\begin{array}{l}\text { BP model meta- } \\
\text { model connectors }\end{array}$ & $\begin{array}{c}\text { Domain Ontology meta- } \\
\text { model relations }\end{array}$ \\
\hline $\begin{array}{l}\text { Sequence Flow } \\
\text { Message Flow }\end{array}$ & Temporal \\
Role & Communication, Transfer \\
& $\begin{array}{l}\text { Execution, Manipulation, } \\
\text { Observation, Influence }\end{array}$
\end{tabular}

Similarly, we have established mappings between meta-model relations of BPM and those of the ontology meta-model. The result is given in Table 2 .

The second type of mapping, presented in the following section, is richer, being based on the semantics of concepts.

\subsection{Semantics based Mapping Rules}

Based on meta-models presented above, we developed a set of matching rules, allowing the mapping of the ontology field with the concepts of process models. These rules are written in Object Constraint Language (OCL) (OMG, 2010).

There are four classes of matching rules. The rules are all defined as functions having as input one or several BP model concepts and returning one or several concepts from the domain ontology.

- Equivalence: returns the ontology concept which is syntactically equivalent (they have the same names) to the BP model concept.

- Synonymy: returns a set of ontology concepts that are synonyms of the BP model concept. The synonymy value is calculated by comparing the existence of common names or synonyms based on Wordnet [25].

- More general: returns the ontology concepts having a superiority relationship (also called hyperonymy or IS-A relationship) with a concept from the ontology already detected as synonym or equivalent to the BP model concept.

- More Specific: returns the ontology concepts having an inferiority relationship with a concept from the ontology detected as synonym or equivalent to the BP model concept.

These classes of rules are instantiated for each of the concepts of the BP metamodel. For each class, an example is given below. 


\subsection{Application to the example}

To illustrate the alignment activity of our approach, we consider the example of "mission order" process. The input is the business process model under construction represented at Figure 1 and an excerpt from the domain ontology "mission plan".

As mentioned above, all the semantic rules are based on type mapping rules i.e. equivalence is between concepts that are not only syntactically equivalent but also type mapped.

Equivalence rules on the actor "financial service" are not fired since the concepts from the ontology do not have the same names as concepts from the BP model of the example. Also we can catch the actions synonym of the process "formalities management" by applying the synonymy rule: synonym(carry out mission formalities) returns "formalities management" and synonym(employee) returns \{staff, internal staff, external staff, missionary, secretary\}. By querying the ontology and firing the semantic/type mapping rules, we can map the ontology concepts to the BPM concepts. Thus we elicit the equivalent, synonyms, different abstraction levels of concepts. But finally, it's up to the analyst to validate these mappings.

The step forward builds on these mappings to detect and correct quality defects. For example the ontology provides several kinds of costs such as "car rental costs" or the distinction between "train" and "flight" tickets that are not considered within the process in hand. This could express an incompleteness of the BP model that could be corrected by replacing "ticket" by "travel costs tickets" if the BP is the same for all the travel costs or it can be redesigned if not. Another enrichment that could be provided thanks to the ontology is the distinction between internal and external staff for which the reimbursement process can be different.

\section{Conclusion}

The quality of business process models is a hot topic both for researchers and practitioners. Many studies demonstrated that the quality of produced models depends highly on the degree of expertise of the modelers. Moreover, modeling activities are practiced by a significant number of non experts including IT professionals. One of the reasons impacting the quality of produced models is the lack of domain knowledge covering both knowledge about the methods and notations used as well as application domain knowledge. In this paper, we tried to propose a solution aiming to exploit application domain knowledge in the improvement of BP models. Our approach considers domain ontologies that are produced in several disciplines (web services, health care, administrative processes etc.) to improve the semantic quality of BP models.

We have defined BP model and ontology meta-models in order to provide a uniform description of both process models and domain ontologies. We have then defined an alignment process using both type-based and semantics-based mappings to detect similarities between concepts from the BP models and the domain ontologies. The results serve as an input for the semantic quality evaluation and improvement processes. 
As regards the alignment process, the future research aims to validate the approach on real case studies based on domain ontologies widely agreed and accepted by practitioners and/or researchers. The rules need also to be completed to cover all the kinds of concepts and relationships semantics. To help in achieving these objectives, we are currently developing a prototype for the definition and execution of mapping rules.

\section{$7 \quad$ References}

1. The International Standards Organisation ISO

2. Vanderfeesten, Irene, Jorge Cardoso, Hajo A Reijers, et Wil Van Der Aalst. «Quality Metrics for Business Process Models ». BPM and Workflow handbook 3 (2006): 1-12.

3. Johansson H.J. et al. (1993), Business Process Reengineering: BreakPoint Strategies for Market Dominance, John Wiley \& Sons

4. Aguilar-Savén R. S., Business process modelling: Review and framework, International Journal of Production Economics, Volume 90, Issue 2, 28 July 2004, ISSN 0925-5273,

5. Becker J, Rosemann M., Uthmann C. V.: Guidelines of Business Process Modeling. Business Process Management 2000: 30-49

6. Mendling J., Recker J. and Reijers H.A. " On the Usage of Labels and Icons in Business Process Modeling" in IJISMD 1(2): 40-58 (2010)

8. Jansen-Vullers M.H. and Netjes M. "Business Process Simulation A Tool Survey" in Workshop and Tutorial on Practical Use of Coloured Petri Nets and the CPN

9. Van der Aalst W. M. P." Challenges in Business Process Analysis" in ICEIS (Selected Papers) 2007: 27-42

10. Vanderfeesten I., Cardoso J., Mendling J., Reijers, Alast: Quality Metrics for Business Process Models. In: Fischer, L. (ed.) BPM and Workflow Handbook 2007 (May 2007).

11. Ghani A.., Muketha K., Wen W.: Complexity Metrics for Measuring the Understandability and Maintainability of Business Process Models using Goal-Question-Metric (GQM). Journal of Computer Science 8, 219-225 (2008).

13. Gruhn V., Laue R.; Complexity metrics for business process models ; 9th international conference on business information systems (BIS 2006).

15. Mendling J., Rejers J. and Cardoso: What Makes Process Models Understandable? In: Lecture Notes in Computer Science, 2007, Volume 4714/2007, 48-63.

16. Krogstie J., Lindland O. I., Sindre G., Defining quality aspects for conceptual models. In IFIP international working conference on Information system concepts. 1995.

17. Eriksson H, Penker M. , Business Modeling with UML, 2000.

18. Beate List and Birgit Korherr 2006. An evaluation of conceptual business process modelling languages. In ACM symposium on Applied computing (SAC '06).

19. Loja, L. F. B., Graciano Neto, V. V., da Costa, S. L., and de Oliveira, J. L. (2010). A business process metamodel for enterprise information systems automatic generation. In Anais do I Congresso Brasileiro de Software.

//da Costa, S. L., Graciano Neto, V. V., Loja, L. F. B., and de Oliveira, J. L. (2010). A

Metamodel for Automatic Generation of Enterprise Information Systems. In Anais do I Congresso Brasileiro de Software: Teoria e Prática - I Workshop Brasileiro de Desenvolvimento de Software Dirigido por Modelos, volume 8, pages 45-52, Salvador, BA, Brasil. UFBA.

22. Purao S. , Storey V. " A multi-layered ontology for comparing relationship semantics in conceptual models of databases. », Journal Applied Ontology archive January 2005

23. Cardoso J. Log-based Complexity of Workflow Patterns, In: Proceedings of the 2007 OTM Confederated international conference On the move to meaningful internet systems. 
24. Khatri V., Vessey I., Ramesh V., Clay P.: Exploring the Role of Application and IS Domain Knowledge Information Systems Research 17(1), pp. 81-99, (C2006 INFORMS

25. Fellbaum C. WordNet: An Electronic Lexical Database. Cambridge, MA: MIT Press. $(1998$, ed.) 